\title{
Novos registros de répteis para as áreas abertas naturais do planalto e do litoral sul de Santa Catarina, Brasil
}

\author{
Tobias Saraiva Kunz ${ }^{1,2 *}$ \\ Ivo Rohling Ghizoni Jr. ${ }^{2}$ \\ Luís Olímpio Menta Giasson² \\ ${ }^{1}$ Programa de Pós-Graduação em Biologia Animal, Departamento de Zoologia \\ Universidade Federal do Rio Grande do Sul \\ Avenida Bento Gonçalves 9500, bloco IV, Prédio 43435, sala 102 \\ CEP 91501-970, Porto Alegre - RS, Brasil \\ ${ }^{2}$ Caipora Cooperativa para Conservação da Natureza \\ Avenida Desembargador Vitor Lima, 260, Sala 513 \\ CEP 88040-400, Florianópolis - SC, Brasil \\ *Autor para correspondência \\ tskunz6@terra.com.br
}

Submetido em 30/11/2010

Aceito para publicação em 26/05/2011

\section{Resumo}

Apresentamos novos registros de répteis para as áreas abertas naturais do planalto e do litoral do estado de Santa Catarina, incluindo os primeiros registros de Teius oculatus e Liophis poecilogyrus sublineatus para este estado e os primeiros registros litorâneos de Atractus reticulatus, Liophis flavifrenatus e Thamnodynastes strigatus. São apresentados, também, novos registros de Xenodon dorbignyi e Oxyrhopus rhombifer para o litoral catarinense e de Liophis jaegeri para o planalto.

Palavras-chave: Campos, Restingas, Squamata, Sul do Brasil

\section{Abstract}

New records of reptiles from natural open areas of the plateau and South coast of Santa Catarina, Brazil. We present new records of reptiles from natural open areas of the plateau and coast of the State of Santa Catarina, including the first records of Teius oculatus and Liophis poecilogyrus sublineatus in this State, as well as the first coastal records of Atractus reticulatus, Liophis flavifrenatus, and Thamnodynastes strigatus. Furthermore, we present new records of Xenodon dorbignyi and Oxyrhopus rhombifer from the coast of Santa Catarina and Liophis jaegeri from the plateau.

Key words: “Campos”, "Restingas”, Squamata, Southern Brazil 


\section{Introdução}

Em Santa Catarina, áreas abertas naturais ocorrem em duas regiões fitoecológicas distintas. Nas porções mais elevadas do planalto, campos aparecem em meio às matas de araucária em manchas de maior ou menor extensão, ocupando grandes áreas nas regiões de Lages e São Joaquim. Já no litoral do estado, as áreas abertas naturais estão presentes em uma estreita faixa de restingas com dunas que se estende desde a Ilha de Santa Catarina, em direção ao sul, até o Uruguai (REITZ, 1961; HUECK; SEIBERT, 1972; KLEIN, 1978; LEITE, 2002; QUADROS; PILLAR, 2002; AB'SABER, 2006). Tanto os campos do planalto quanto a faixa litorânea de restingas com dunas estão inseridos no domínio morfoclimático do bioma Mata Atlântica (IBGE, 2004).

O conhecimento sobre a fauna de répteis do estado de Santa Catarina ainda é incipiente, baseado em contribuições pontuais na literatura e concentrado em áreas florestais (e.g. MULLER; RITTER, 1978; BÉRNILS et al., 2000; 2001; MARQUES et al., 2001; HARTMANN; GIASSON, 2008; KUNZ; GHIZONI JR., 2009). Contudo, recentemente Ghizoni Jr. et al. (2009) apresentaram registros para 16 espécies de répteis de áreas abertas do planalto e do litoral, anteriormente desconhecidas ou com poucos registros para o estado. Através de atividades de campo recentes e esforço de procura direcionado às áreas abertas naturais (campos e restingas) de Santa Catarina, obtivemos novos e relevantes registros de répteis para estas formações.

\section{Material e Métodos}

Os registros foram obtidos durante atividades de campo recentes, incluindo inventários para diagnósticos ambientais realizados entre 2009 e 2010 nas áreas de predomínio das formações abertas no estado de Santa Catarina, mas também através do exame dos exemplares depositados na coleção herpetológica do Departamento de Ecologia e Zoologia da Universidade Federal de Santa Catarina (CHUFSC).

Para todas as localidades com registros inéditos são fornecidas coordenadas geográficas (latitude/ longitude) e altitude do topônimo (quando no planalto).
Os exemplares coletados foram depositados nas coleções herpetológicas CHUFSC e do Departamento de Zoologia da Universidade Federal do Rio Grande do Sul (UFRGS).

\section{Resultados e Discussão}

Obtivemos os primeiros registros de Teius oculatus (D'ORBIGNY; BIBRON, 1837) e Liophis poecilogyrus sublineatus (COPE, 1860) para Santa Catarina, e os primeiros registros de Atractus reticulatus (BOULENGER, 1885), Liophis flavifrenatus (COPE, 1862) e Thamnodynastes strigatus (GÜNTHER, 1858) para o litoral catarinense. Também apresentamos novos registros e localidades para Oxyrhopus rhombifer Duméril, Bibron \& Duméril, 1854 e Xenodon dorbignyi (DUMÉRIL; BIBRON; DUMÉRIL, 1854) para o litoral e uma nova localidade para Liophis jaegeri (GÜNTHER, 1858) no planalto (Figura 1).

A seguir são apresentados e discutidos os registros para cada espécie, incluindo foto dos exemplares, sempre que possível.

\section{Ordem Squamata}

Família Teiidae

Teius oculatus (D’ORBIGNY; BIBRON, 1837)

Espécie conhecida para os estados do Rio Grande do Sul e Paraná, além de Uruguai, Paraguai e Argentina (LEMA, 1994; BÉRNILS et al., 2007; CAPPELLARI et al., 2007). No Rio Grande do Sul a espécie é conhecida apenas para áreas baixas, onde ocupa desde restingas até formações rochosas em morros de baixa elevação (CAPPELLARI et al., 2007). Alguns indivíduos foram observados, por IRG e LOMG, ao longo de um trecho da rodovia BR-470 e suas margens no município de

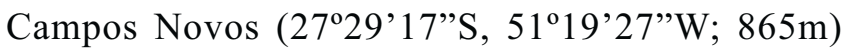
em 18/XI/2009. Um indivíduo (CHUFSC, 1212) foi capturado após entrar em toca de pouca profundidade. No dia seguinte um indivíduo (CHUFSC 1213) foi encontrado atropelado no mesmo local. Em 04/XII/2009, novamente foram observados diversos indivíduos, por IRG e TSK, sendo coletado um exemplar (UFRGS 5562; Figura 2). Aparentemente essa população está confinada às margens e à faixa de domínio da rodovia, 


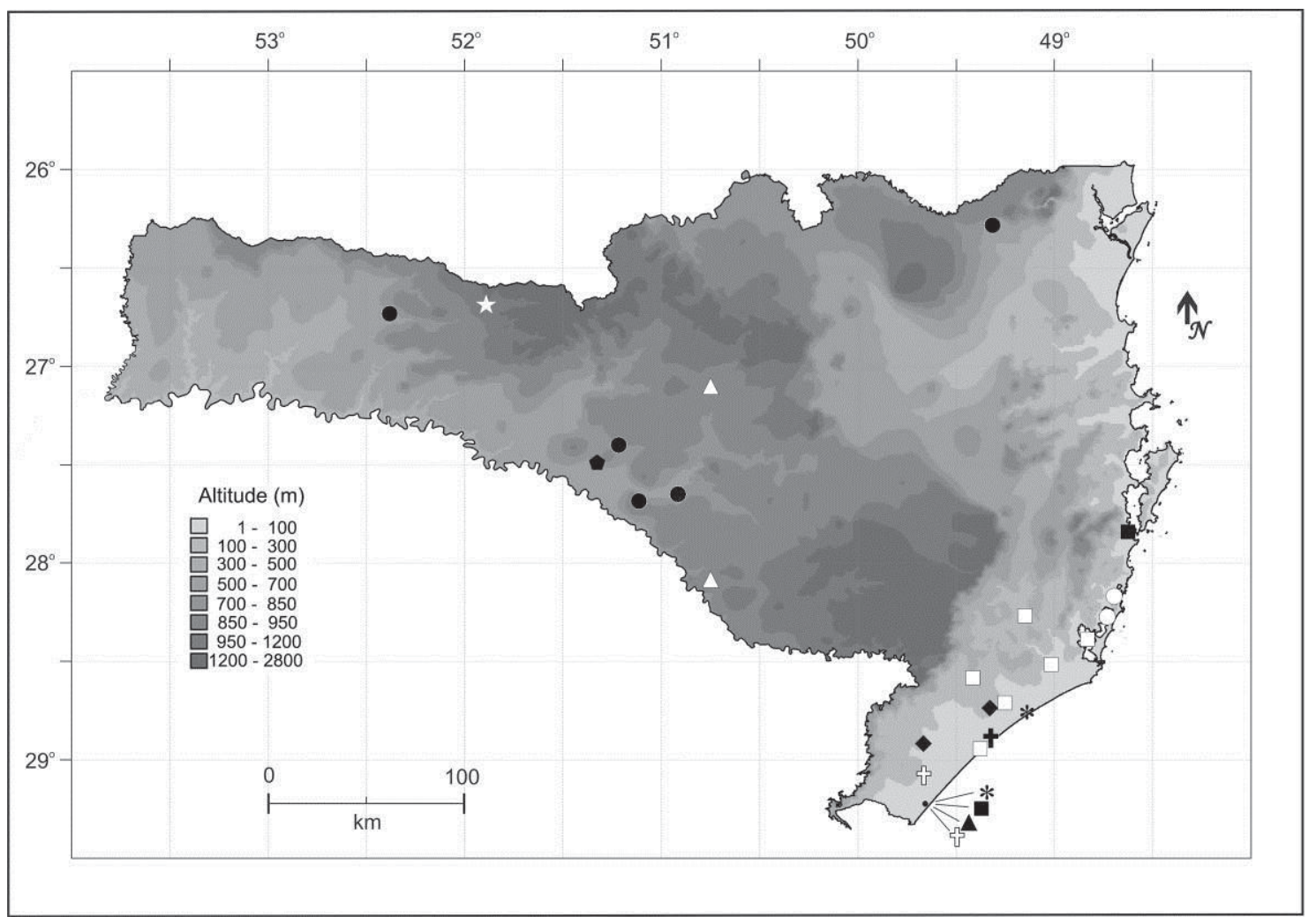

FIGURA 1: Mapa do estado de Santa Catarina indicando os pontos de registro dos répteis. Círculos: Atractus reticulatus, círculos brancos: presente trabalho, círculos pretos: registros feitos por Ghizoni Jr. et al. (2009); quadrados: Oxyrhopus rhombifer, quadrados brancos: registros litorâneos apresentados por Ghizoni Jr. et al. (2009), quadrados pretos: presente trabalho; triângulos: Liophis flavifrenatus, triângulos brancos: registros feitos por Ghizoni Jr. et al. (2009), triângulo preto: presente trabalho; cruzes: Xenodon dorbignyi, cruzes brancas: registros feitos por Ghizoni Jr. et al. (2009), cruz preta: presente trabalho; losangos pretos: Thamnodynastes strigatus; asteriscos: Liophis poecilogyrus sublineatus; pentágono preto: Teius oculatus; estrela branca: Liophis jaegeri. Para T. strigatus e O. rhombifer foram exibidos apenas os registros litorâneos.

onde diversas tocas foram observadas. Mesmo quando perseguidos, nenhum indivíduo procurou refúgio nas extensas plantações de soja e milho imediatamente adjacentes à faixa de domínio.

\section{Família Dipsadidae}

\section{Atractus reticulatus (BOULENGER, 1885)}

Atractus reticulatus ocorre desde o estado de Minas Gerais para o sul até o norte do Uruguai, no departamento de Rivera e na província de Corrientes, na Argentina, ocupando áreas de campo, pampa e cerrado, além de zonas de transição entre essas formações e áreas de floresta estacional (PASSOS et al., 2010). Em Santa Catarina, até o momento era conhecida apenas para o planalto (GHIZONI JR. et al., 2009).
Em 09/IX/2009 dois indivíduos (CHUFSC 1200, UFRGS 5563) foram encontrados por TSK sob entulho em área de restinga a apenas $50 \mathrm{~m}$ da faixa de areia da praia da Vila, em Imbituba (28 $16^{\circ} 04^{\prime}$ 'S, 48 41'09'W). Em 06/IV/2010 um juvenil foi encontrado (CHUFSC 1235) sob tronco, por IRG, no mesmo município (2812'44”S, 4841'05'W) (Figura 3).

\section{Liophis jaegeri (GÜNTHER, 1858)}

Liophis jaegeri é uma espécie semi-aquática, geralmente associada a áreas alagadas em ambientes abertos (BORGES-MARTINS et al., 2007). Embora seja uma serpente amplamente distribuída, conhecida para o sul, sudeste e centro-oeste do Brasil, além de Argentina, Uruguai e Paraguai, e de ter sido a segunda espécie mais frequente em estudo desenvolvido no extremo nordeste 


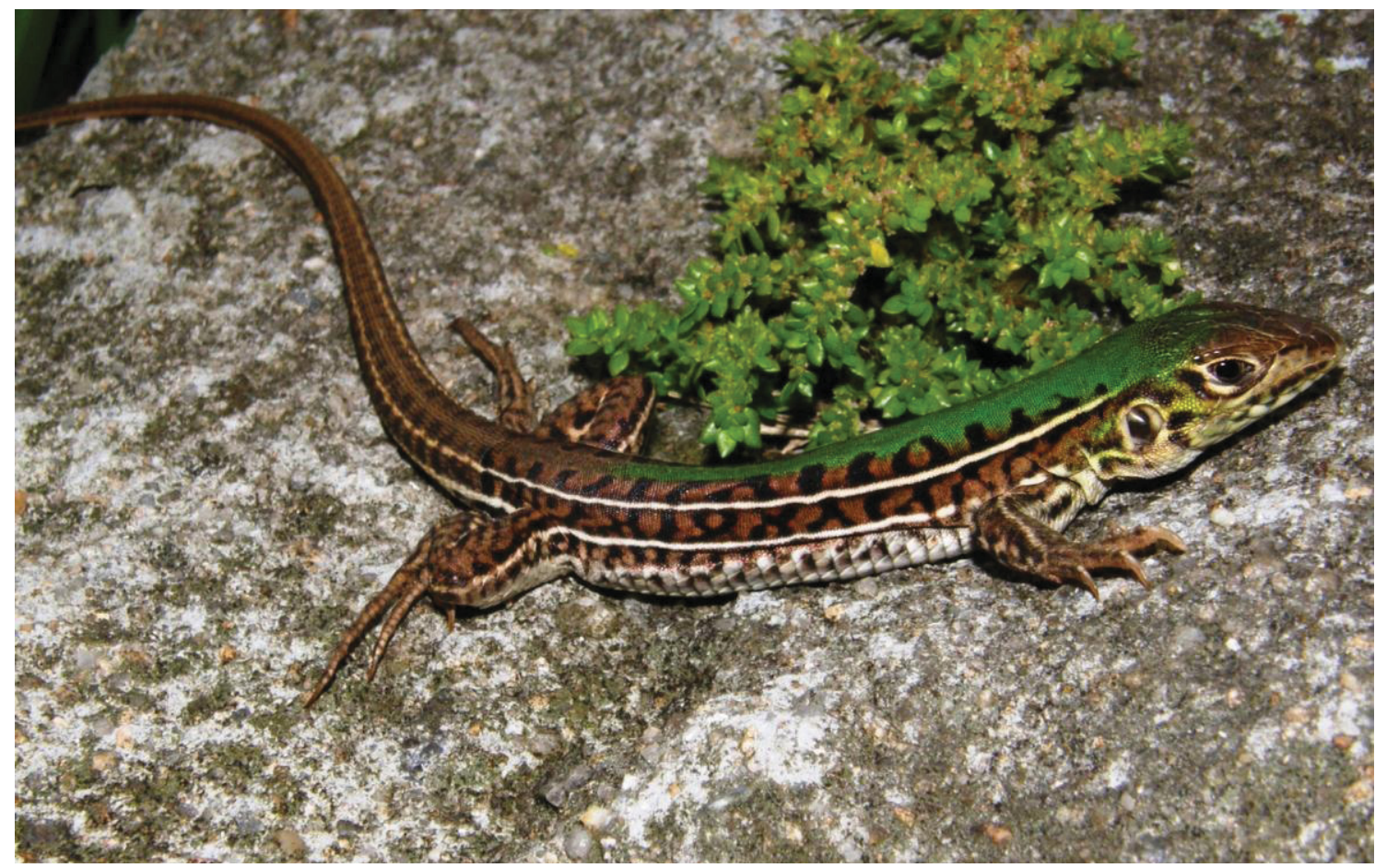

FIGURA 2: Teius oculatus (UFRGS 5562), Campos Novos, Santa Catarina.

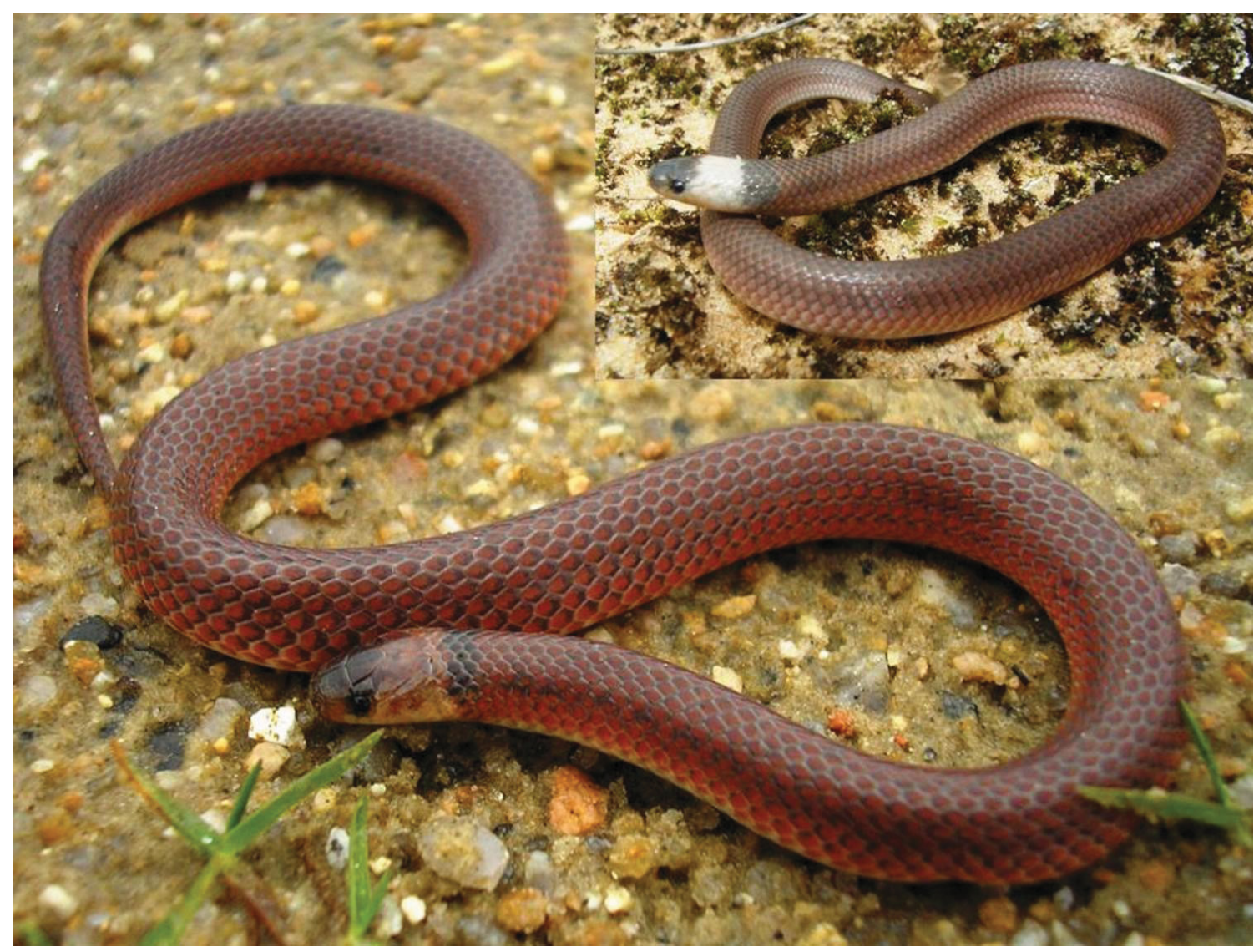

FIGURA 3: Atractus reticulatus, praia da Vila, Imbituba, Santa Catarina. No detalhe, exemplar juvenil (CHUFSC 1235) do mesmo município. 
do planalto do Rio Grande do Sul (DI-BERNARDO et al., 2007), pouco se sabe sobre sua ocorrência em Santa Catarina. Bérnils et al. (2001) citou a espécie para o Alto Vale do Itajaí e, de acordo com os mapas de distribuição apresentados por Dixon $(1987 ; 1989)$, a espécie ocorreria em todo o meio-oeste catarinense.

Em 29/XI/2010, um exemplar (CHUFSC 1327) foi encontrado morto por Maicon F. Silva na área do canteiro de obras da Pequena Central Hidrelétrica Passos Maia, às margens do rio Chapecó (2640'58'S, 5153'29'W, 1080m), no município de Passos Maia.

\section{Liophis poecilogyrus sublineatus (COPE, 1860)}

Liophis poecilogyrus é uma espécie amplamente distribuída na América do Sul à leste dos Andes, desde a bacia amazônica até a Argentina (DIXON, 1989), e de taxonomia complexa. Em Santa Catarina, há registros apenas para o planalto, no oeste do estado (HARTMANN; GIASSON, 2008; KUNZ; GHIZONI
JR., 2009), onde ocorre a forma Liophis poecilogyrus schotti (GIRAUDO, 2001; GIRAUDO; SCROCCHI, 2002). Liophis poecilogyrus sublineatus é a forma pampeana, com registros para Argentina, Uruguai e Rio Grande do Sul (GIRAUDO; SCROCCHI, 2002) e foi citada como de possível ocorrência para o litoral sul catarinense (GHIZONI JR. et al., 2009).

Em 22/XI/2009, um indivíduo (CHUFSC 1216) foi encontrado morto, possivelmente atropelado, no balneário Esplanada, município de Jaguaruna (28 45'38'S, 4907'50"W), por TSK. Um juvenil (UFRGS 5627; Figura 4) foi encontrado sob entulhos, em 24/III/2010, por TSK, no município de Balneário

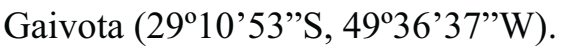

\section{Liophis flavifrenatus (COPE, 1862)}

Esta espécie ocorre em áreas abertas naturais desde São Paulo e Mato Grosso do Sul até o Rio Grande do Sul, Uruguai e norte da Argentina, e foi registrada

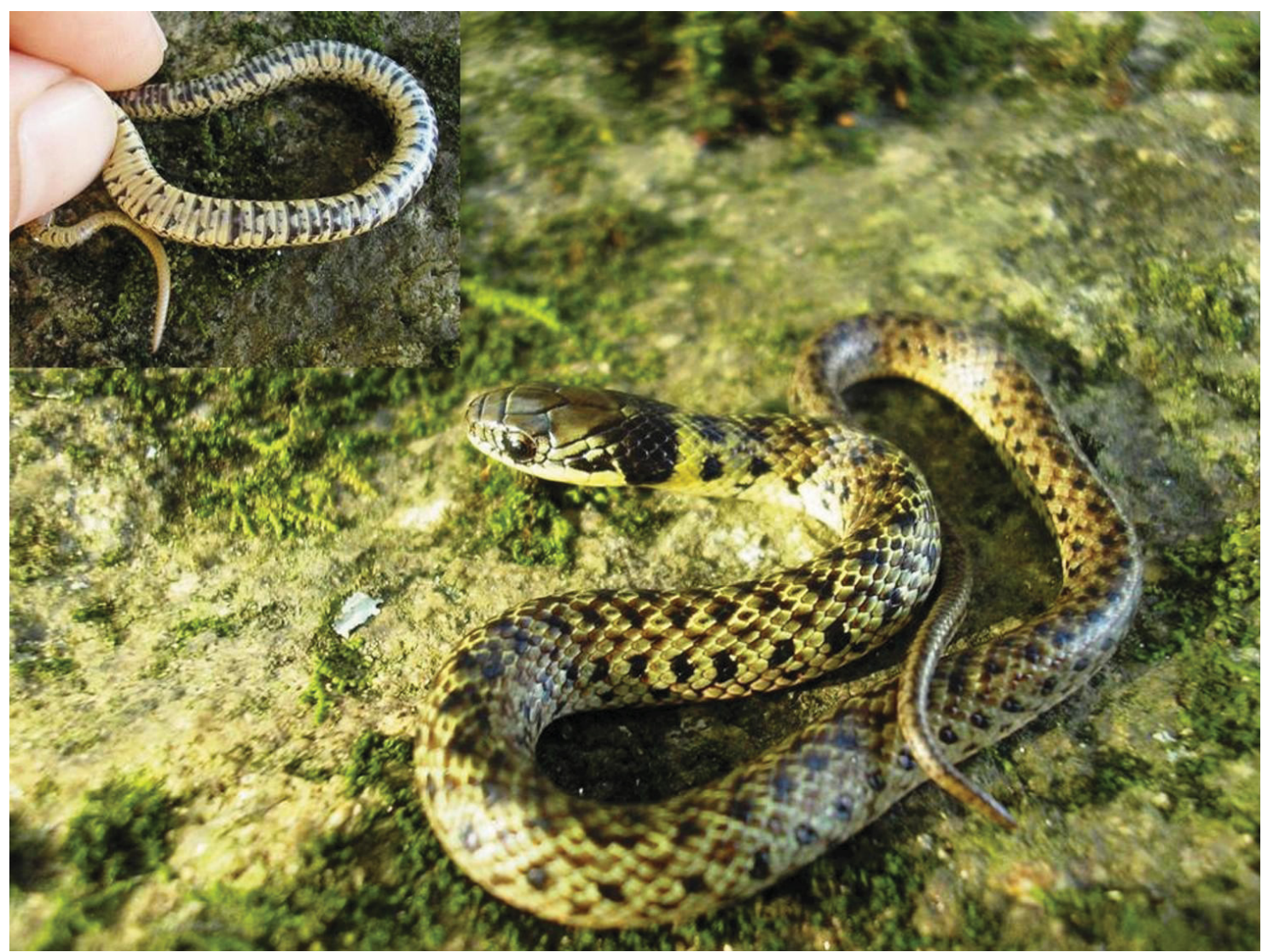

FIGURA 4: Liophis poecilogyrus sublineatus (UFRGS 5627), Balneário Gaivota, Santa Catarina. No detalhe, vista ventral do mesmo indivíduo. 


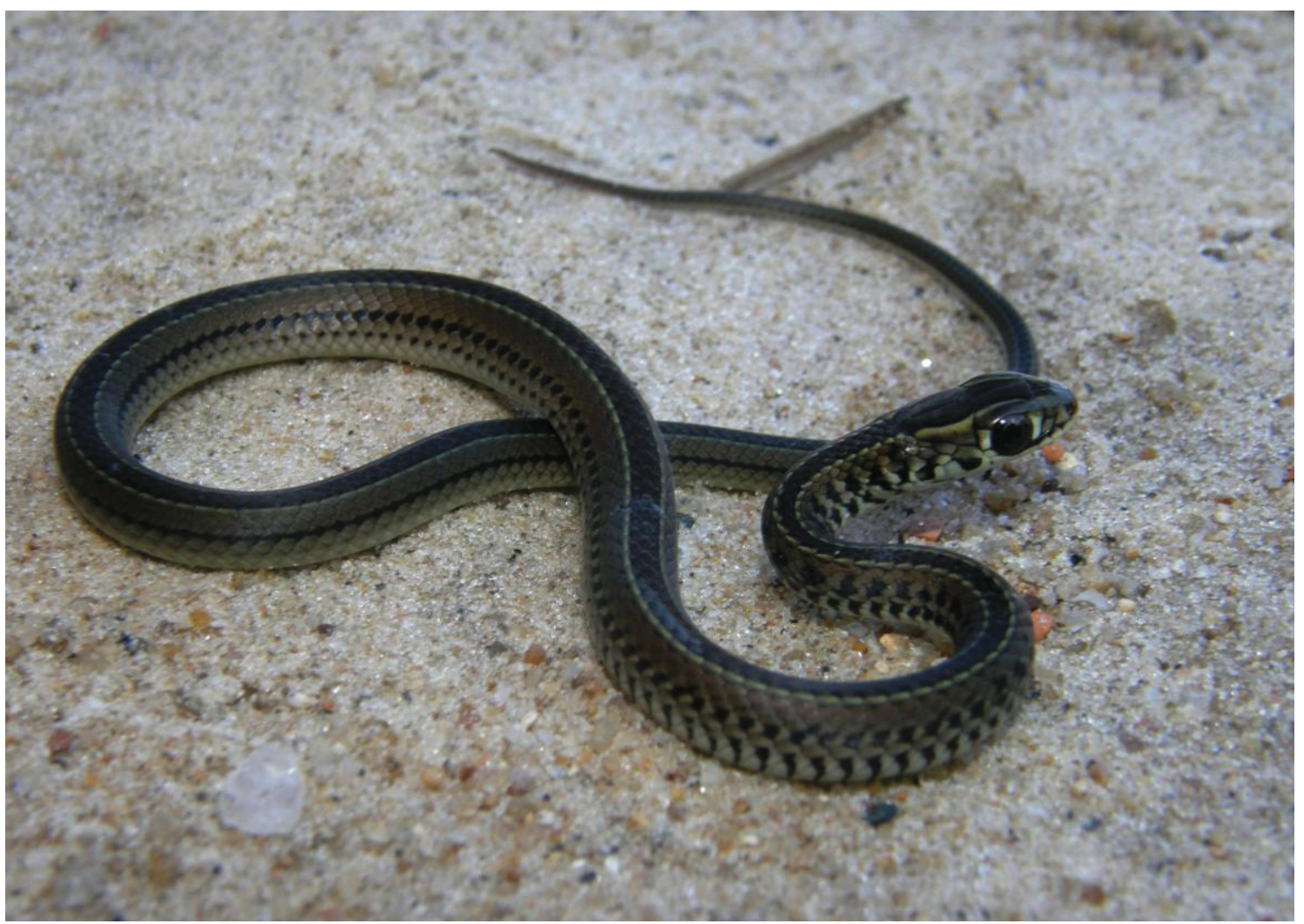

FIGURA 5: Liophis flavifrenatus (CHUFSC 1251), Balneário Gaivota, Santa Catarina.

recentemente para o planalto catarinense com base em registros de coleção (GHIZONI JR. et al., 2009). Em 26/IV/2010, um indivíduo juvenil (CHUFSC, 1251; Figura 5) foi encontrado sob entulhos, ao lado de um indivíduo também juvenil de Oxyrhopus rhombifer, por TSK, no município de Balneário Gaivota (29¹0'53”S, 493' $37^{\prime \prime} \mathrm{W}$ ). 1854

Oxyrhopus rhombifer Duméril, Bibron \& Duméril,

Oxyrhopus rhombifer ocorre no Brasil desde o sul do rio Amazonas até o Rio Grande do Sul, Uruguai e Argentina. Em Santa Catarina é conhecida para o planalto e para o litoral sul, sendo citada nesta última região para Araranguá, Braço do Norte, Içara, Laguna, Siderópolis e Tubarão (GHIZONI JR. et al., 2009; KUNZ; GHIZONI JR., 2009).

Um indivíduo juvenil (Figura 6) foi encontrado por TSK sob entulhos (Figura 7), ao lado de juvenil de Liophis flavifrenatus, no município de Balneário Gaivota (29 $\left.10^{\circ} 53^{\prime \prime} \mathrm{S}, 49^{\circ} 36^{\prime} 37^{\prime \prime} \mathrm{W}\right)$, a menos de $20 \mathrm{~km}$ da divisa com o estado do Rio Grande do Sul. Outro indivíduo (CHUFSC 1232) foi encontrado morto por Marcos A. Tortato na Baixada do Maciambu, município de Palhoça (2750'30"S, 48 37'30”'W), limite norte das formações contínuas de restingas com dunas no continente de Santa Catarina (essas formações ocorrem mais ao norte apenas na Ilha de Santa Catarina). Juntos, esses registros evidenciam a amplitude da distribuição da espécie na porção sul da planície litorânea catarinense.

\section{Thamnodynastes strigatus (GÜNTHER, 1858)}

Thamnodynastes strigatus é uma serpente amplamente distribuída no sul e sudeste do Brasil, na Argentina, no Paraguai e no Uruguai. No sul do Brasil é conhecida para o planalto, nos estados do Paraná, Santa Catarina e Rio Grande do Sul, neste último ocorrendo também em áreas baixas (Pampa) (FRANCO; FERREIRA, 2002). Em Santa Catarina havia registros apenas para a região oeste (planalto) (HARTMANN; GIASSON, 2008; KUNZ; GHIZONI JR., 2009). 


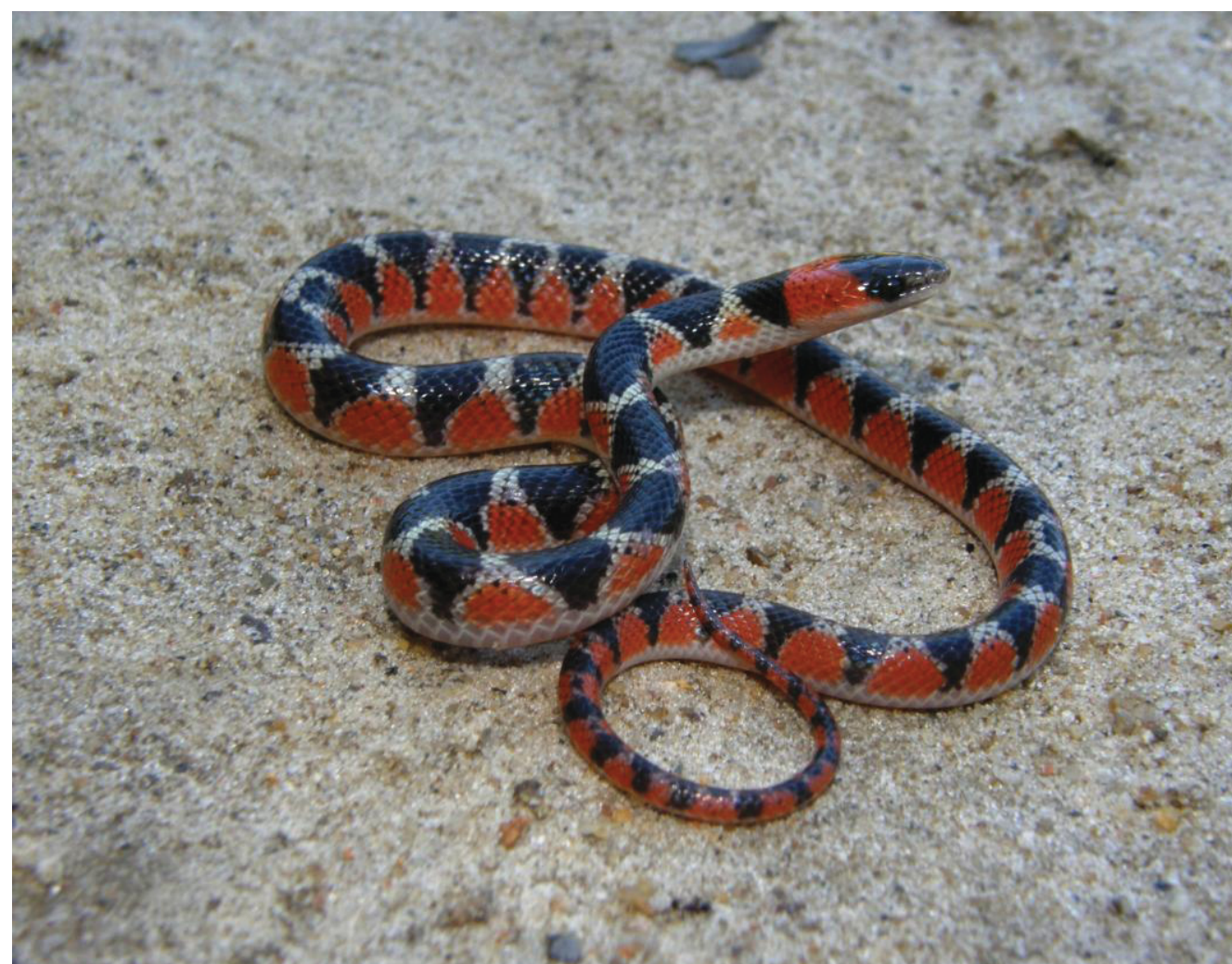

FIGURA 6: Oxyrhopus rhombifer, Balneário Gaivota, Santa Catarina.

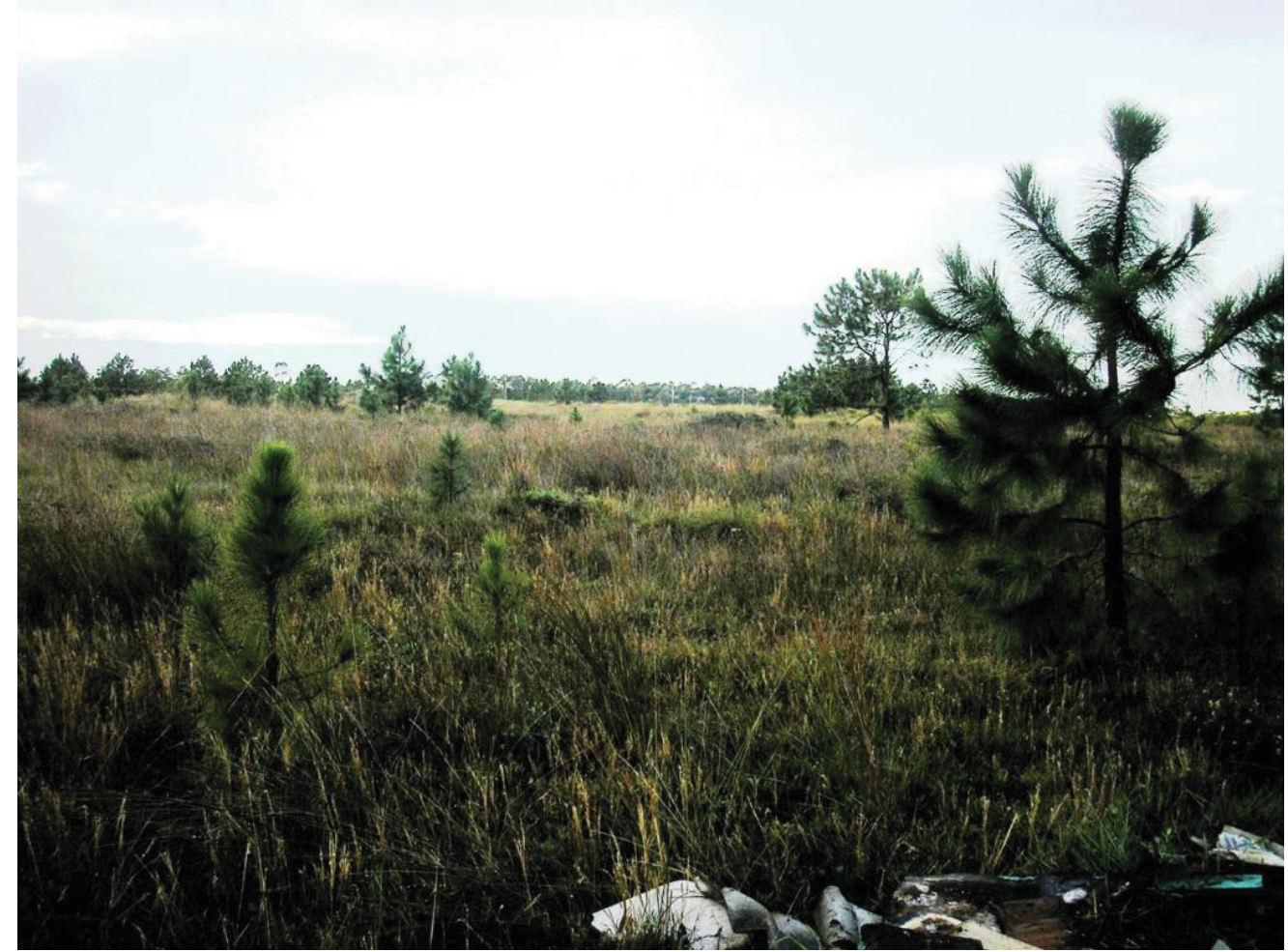

FIGURA 7: Local onde foram coletados exemplares de Liophis flavifrenatus, Liophis poecilogyrus sublineatus e Oxyrhopus rhombifer (sob os entulhos que aparecem no canto inferior direito da imagem), no município de Balneário Gaivota, Santa Catarina. 
Um indivíduo (UFRGS 5601; Figura 8) foi encontrado por TSK em área de restinga com dunas e banhados, na margem esquerda do rio Araranguá (285'13”S, 49¹9'29”'W), no município de Araranguá. Em coleção, foram obtidos mais dois registros (CHUFSC $718,878)$, ambos para o município de Turvo $\left(28^{\circ} 55^{\prime} \mathrm{S}\right.$, $\left.49^{\circ} 40^{\prime} \mathrm{W}\right)$.

Xenodon dorbignyi (DUMÉRIL; BIBRON; DUMÉRIL, 1854)

Xenodon dorbignyi ocorre nos estados de Santa Catarina e Rio Grande do Sul, na Argentina, no Paraguai e no Uruguai. Em Santa Catarina era conhecida por citação na descrição original, sem uma localidade precisa no estado (OREJAS-MIRANDA, 1966) e por apenas mais dois exemplares colecionados nos municípios de Passo de Torres e Sombrio, no extremo sul do litoral catarinense (GHIZONI JR. et al., 2009).

Em 26/VI/2009, um exemplar (CHUFSC 1214) foi encontrado atropelado em estrada secundária, no município de Araranguá (2854’13”S, 49²0’36”W), por TSK. Este registro amplia em aproximadamente $36 \mathrm{~km}$ ao norte a distribuição conhecida da espécie no estado.

De acordo com os padrões de distribuição apresentado por Ghizoni Jr. et al. (2009) para os répteis de áreas abertas do sul do Brasil, T. oculatus, A. reticulatus, L. flavifrenatus, L. jaegeri, O. rhombifer e T. strigatus se enquadram entre as espécies que ocupam tanto as áreas abertas do planalto quanto das planícies. Dentre estas espécies, A. reticulatus, L. flavifrenatus e T. strigatus contavam com registros em Santa Catarina apenas para o planalto. Teius oculatus não contava com registros para o estado, mas sua ocorrência era esperada, pois há registros para áreas abertas de planície no Rio Grande do Sul e para o planalto paranaense (LEMA, 1994; BÉRNILS et al., 2007; CAPPELLARI et al., 2007). Por sua vez, Liophis poecilogyrus sublineatus, juntamente com Anops kingii Bell 1833, Cnemidophorus lacertoides Duméril \& Bibron, 1839, Bothropoides pubescens (COPE, 1870) e Xenodon dorbignyi, representam espécies com distribuição apenas

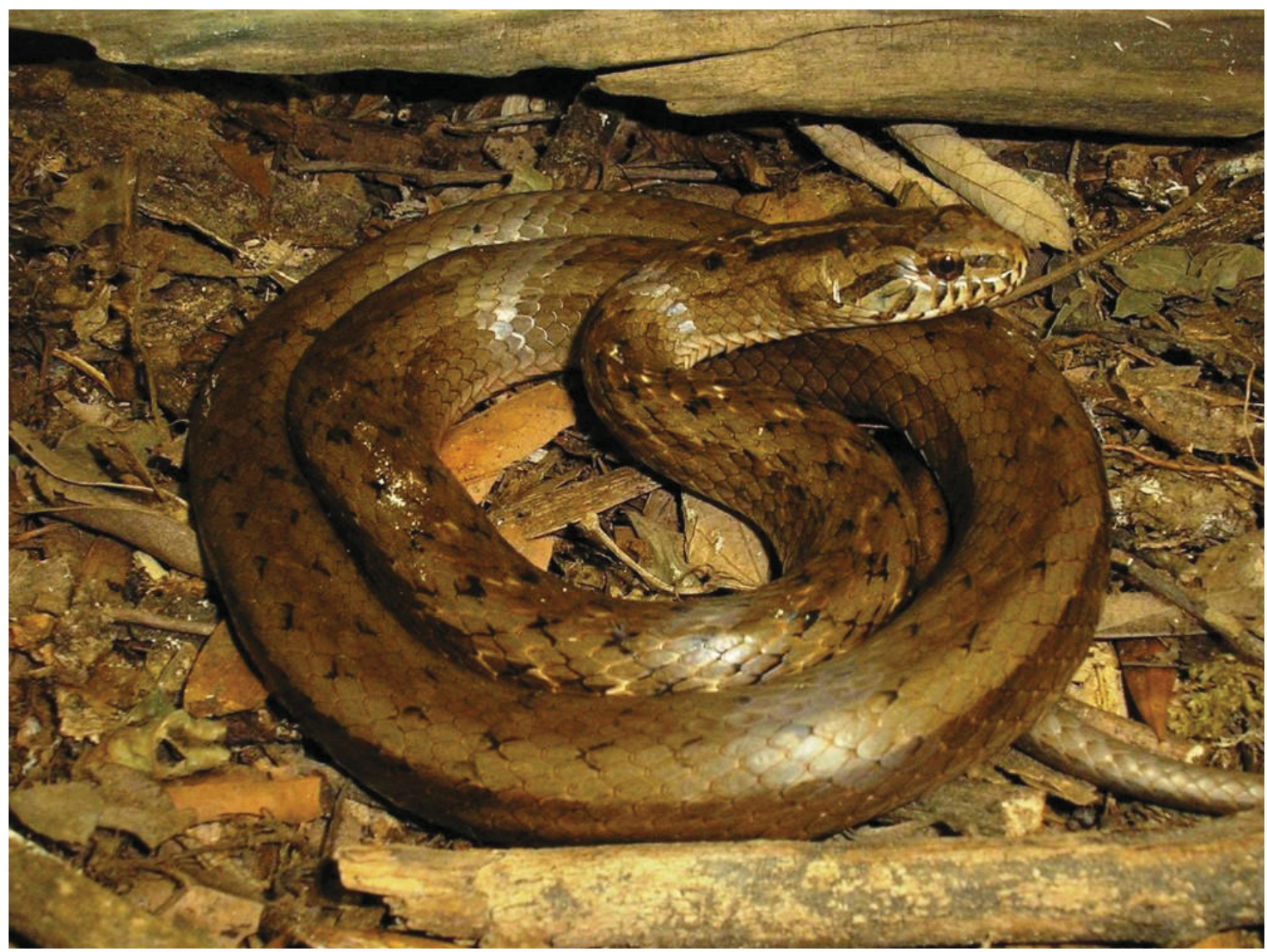

FIGURA 8: Thamnodynastes strigatus (UFRGS 5601), Araranguá, Santa Catarina. 
nas áreas abertas de planície, podendo ser consideradas espécies tipicamente pampeanas, cuja presença em Santa Catarina representa uma continuidade de distribuição de elementos deste bioma para o litoral sul catarinense. Neste sentido, cabe destacar que as formações pioneiras da planície costeira do estado do Rio Grande do Sul foram incluídas no bioma Pampa, coincidindo o limite deste bioma com o limite político com o estado de Santa Catarina (IBGE, 2004).

Entretanto, segundo a abordagem biogeográfica de Cabrera e Willink (1980), os campos litorâneos de Santa Catarina estão localizados na província biogeográfica Atlântica, bem como os campos litorâneos do norte do estado do Rio Grande do Sul. As análises florísticas e fitogeográficas da porção meridional do estado de Santa Catarina, ainda não são suficientemente estudadas e as diferentes classificações propostas, por analisarem a distribuição da vegetação em escalas muito grandes, não reconhecem a continuidade e similaridade com as formações campestres mais austrais. Contudo, os campos litorâneos do sul do estado e sua flora peculiar, se estendem em direção ao norte de forma contínua por cerca de $120 \mathrm{~km}$ até o município de Laguna, a partir de onde passam a ser interrompidos pelos maciços rochosos que se projetam em direção ao oceano, e que apresentam uma predominância de florestas e seus elementos faunísticos típicos (GHIZONI JR. et al., 2009).

Apesar da falta de reconhecimento desse complexo de campos litorâneos em sua totalidade, na qual o estado catarinense não está ainda representado de maneira satisfatória, Suguio e Tessler (1984), estudando a origem e nomenclatura das planícies litorâneas brasileiras, reconheceram para o sul do Brasil uma unidade litorânea entre a região de Laguna (Santa Catarina) e a desembocadura do Arroio Chuí (Rio Grande do Sul). O reconhecimento dessa unidade geomorfológica para a região sugere principalmente que a origem dos eventos paleoecológicos a que fora submetida durante as transições climáticas do Pleistoceno e Holoceno foi a mesma, sendo que a ocupação pela vegetação e, consequentemente, pela fauna terrestre, aconteceram de maneira semelhante. Contudo, a natureza da similaridade da formação vegetacional campestre pampeana e sulina com aquela do litoral catarinense ainda é uma lacuna importante dentro do conhecimento biogeográfico regional. Estudos que abordem outras formas de vida, principalmente a evolução da vegetação no litoral sul do estado de Santa Catarina, poderão esclarecer e aumentar o conhecimento sobre as formações campestres que ali ocorrem.

Por outro lado, os campos do planalto, também incluídos no bioma Mata Atlântica, formam naturalmente um mosaico floresta-campo ao longo do planalto sulbrasileiro e apresentam composição florística e faunística bastante distinta daquela dos ambientes florestais adjacentes, que é compartilhada mais claramente com outros biomas como Pampa e Cerrado. Até recentemente, pesquisadores questionavam se a presença de campos no sul do Brasil não seria decorrente de atividades humanas pré e pós-colombianas (ver BEHLING et al., 2009). Atualmente, a presença de campos em um clima úmido é explicada pela expansão relativamente recente das florestas, sendo os campos o tipo de vegetação mais antigo, representando formações relictuais de um passado onde a região apresentava clima mais seco (QUADROS; PILLAR, 2002; BEHLING et al., 2009). A presença de espécies da fauna campestre, tais como Philodryas agassizii (Répteis: Dipsadidae), Cnemidophorus vacariensis, Teius oculatus (Répteis: Teiidae), Scinax squalirostris e Hypsiboas leptolineatus (Anfíbios: Hylidae) (GHIZONI JR. et al., 2009; este estudo), incapazes de penetrar o ambiente florestal, mesmo em pequenas manchas campestres totalmente circundadas por uma matriz florestal, não deixa dúvidas sobre a origem natural destas formações.

Embora os campos do sul do Brasil estejam atualmente distribuídos em dois biomas distintos (Pampa e Mata Atlântica), estes ecossistemas, sejam de planalto ou de planície, têm sido considerados em conjunto (como "campos sulinos") na elaboração de estratégias de conservação (ver MMA, 2000; PILLAR et al., 2009).

\section{Agradecimentos}

Somos gratos a Anderson S. de Mello por valiosas contribuições ao texto na discussão sobre a fitogeografia dos campos; a ETS e Caruso-Jr Estudos Ambientais \& Engenharia pela logística em parte dos trabalhos de campo; a Marcos A. Tortato pelas informações sobre o exemplar de 
Oxyrhopus rhombifer da Baixada do Maciambu; e a Jorge J. Cherem e Maicon F. Silva pela ajuda em alguns trabalhos de campo. TSK agradece à CAPES pela bolsa concedida.

\section{Referências}

AB'SABER, A. N. Fundamentos da geomorfologia costeira do Brasil atlântico inter e subtropical. In: AB'SABER, A. N. (Ed.). Brasil: Paisagens de exceção. Cotia: Ateliê Editorial, 2006. p. 79-119.

BEHLING， H.; JESKE-PIERUSCHKA， V.; SCHÜLER， L.; PILLAR, V. P. Dinâmica dos campos no sul do Brasil durante o Quaternário Tardio. In: PILLAR, V. P.; MÜLLER, S. C.; CASTILHOS, Z. M. S.; JACQUES, A. V. A. (Eds). Campos Sulinos - conservação e uso sustentável da biodiversidade. Brasília: Ministério do Meio Ambiente, 2009. p. 13-25.

BÉRNILS, R. S.; BATISTA, M. A.; BERTELLI, P. W. Cobras e lagartos do Vale: levantamento das espécies de Squamata (Reptilia, Lepidosauria) da bacia do Rio Itajaí, Santa Catarina, Brasil. Revista de Estudos Ambientais, Blumenau, v. 3, n. 1, p. 69-79, 2001.

BÉRNILS, R. S.; GIRAUDO, A. R.; CARREIRA, S.; CECHIN, S. Z. Répteis das porções subtropical e temperada da região Neotropical. Ciência \& Ambiente, Santa Maria, v. 35, p. 101-136, 2007.

BÉRNILS, R. S.; MORATO, S. A. A.; MOURA-LEITE, J. C. Imantodes cenchoa (Dormideira). Geographic distribution. Herpetological Review, St. Louis, v. 31, n. 1, p. 55-56, 2000.

BORGES-MARTINS, M.; ALVES, M. L. M.; ARAUJO, M. L.; OLIVEIRA, R. B.; ANÉS, A. C. Répteis. In: BECKER, F. G.; RAMOS, R. A.; MOURA, L. A. (Orgs). Biodiversidade: Regiões da Lagoa do Casamento e dos Butiazais de Tapes, Planície Costeira do Rio Grande do Sul. Brasília: Ministério do Meio Ambiente, 2007. p. 292-315.

CABRERA, A. L.; WILLINK, A. Biogeografia de América Latina. 2 ed. Washington: OEA, 1980. 117 p.

CAPPELLARI, L. H.; LEMA, T.; PRATES JR., P.; ROCHA, C. F. D. Diet of Teius oculatus (Sauria, Teiidae) in southern Brazil (Dom Feliciano, Rio Grande do Sul). Iheringia, Série Zoologia, Porto Alegre, v. 97, n. 1, p. 31-35, 2007.

DI-BERNARDO, M.; BORGES-MARTINS, M.; OLIVEIRA, R. B.; PONTES, G. M. F. Taxocenoses de serpentes de regiões temperadas do Brasil. In: NASCIMENTO, L. B.; OLIVEIRA, M. E. (Eds.). Herpetologia no Brasil II. Belo Horizonte: Sociedade Brasileira de Herpetologia, 2007. p. 222-263.

DIXON, J. R. A key and checklist to the neotropical snake genus Liophis with country lists and maps. Smithsonian Herpetological Information Service, Washington, v. 79, p. 1-40, 1989.

DIXON, J. R. Taxonomy and geographic variation of Liophis typhlus and related "green" species of South America (Serpentes: Colubridae). Annals of Carnegie Museum, Pittsburgh, v. 56, n. 8, p. 173-191, 1987.

FRANCO, F. L.; FERREIRA, T. G. Descrição de uma nova espécie de Thamnodynastes Wagler, 1830 (Serpentes, Colubridae) do nordeste brasileiro, com comentários sobre o gênero. Phyllomedusa, Belo Horizonte, v. 1, n. 2, p. 57-74, 2002.

GHIZONI JR., I. R.; KUNZ, T. S.; CHEREM, J. J.; BÉRNILS, R. $\mathrm{S}$. Registros notáveis de répteis de áreas abertas naturais do planalto e litoral do Estado de Santa Catarina, sul do Brasil. Biotemas, Florianópolis, v. 22, n. 3, p. 129-141, 2009.

GIRAUDO, A. R. Serpientes de la selva paranaense y del Chaco Húmedo. Buenos Aires: L.O.L.A., 2001. 328 p.

GIRAUDO, A. R.; SCROCCHI, G. J. Argentinian snakes: an annotated checklist. Smithsonian Herpetological Information Service, Washington, v. 132, p. 1-53, 2002.

HARTMANN, P. A.; GIASSON, L. O. M. Répteis. In: CHEREM, J. J.; KAMMERS, M. (Orgs). A fauna das áreas de influência da Usina Hidrelétrica Quebra-Queixo. Erechim: Habilis Editora, 2008. p. 111-124.

HUECK, K.; SEIBERT, P. Vegetationskarte von Sudamerika. Stuttgart: Gustav Fischer, 1972. 69 p.

IBGE. Mapa da vegetação do Brasil e mapa de biomas do Brasil. Rio de Janeiro: Instituto Brasileiro de Geografia e Estatística, 2004.

KLEIN, R. M. Mapa fitogeográfico do estado de Santa Catarina. Flora Ilustrada Catarinense, parte V: Mapa fitogeográfico. Itajaí: Herbário "Barbosa Rodrigues", 1978. 24 p.

KUNZ, T. S.; GHIZONI JR., I. R. Serpentes encontradas mortas em rodovias do estado de Santa Catarina, Brasil. Biotemas, Florianópolis, v. 22, n. 2, p. 91-103, 2009.

LEITE, P. F. Contribuição ao conhecimento fitoecológico do sul do Brasil. Ciência \& Ambiente, Santa Maria, n. 24, p. 51-73, 2002.

LEMA, T. Lista comentada dos répteis ocorrentes no Rio Grande do Sul, Brasil. Comunicações do Museu de Ciência e Tecnologia da PUCRS, Série Zoologia, Porto Alegre, v. 7, p. 41-150, 1994.

MARQUES, O. A. V.; ETEROVIC, A.; SAZIMA, I. Serpentes da Mata Atlântica. Guia ilustrado para a Serra do Mar. Ribeirão Preto: Holos, 2001. 184 p.

MMA. Avaliação e ações prioritárias para a conservação da biodiversidade da Mata Atlântica e Campos Sulinos. Brasília: Ministério do Meio Ambiente/Secretaria de Biodiversidade e Florestas, 2000. 40.

MÜLLER, P.; RITTER, C. Erstnachweis von Uromacerina ricardinii (Peracca 1897) für den staat Von Santa Catarina (Brasilien) (Reptilia: Serpentes: Colubridae). Salamandra, Frankfurt, v. 14, n. 1, p. 44, 1978.

OREJAS-MIRANDA, B. R. The snake genus Lystrophis in Uruguay. Copeia, Lawrence, v. 1966, n. 2, p. 193-205, 1966.

PASSOS, P.; FERNANDES, R.; BÉRNILS, R. S.; MOURALEITE, J. C. Taxonomic revision of the Brazilian Atlantic Forest Atractus (Reptilia: Serpentes: Dipsadidae). Zootaxa, Auckland, v. 2364, p. 1-63, 2010.

PILlAR, V. P.; MÜlleR, S. C.; CASTILHOS, Z. M. S.; JACQUES, A. V. A. (Eds). Campos Sulinos - conservação e uso sustentável da biodiversidade. Brasília: Ministério do Meio Ambiente, 2009. 403 p.

QUADROS, F. L. F.; PILLAR, V. P. Transições floresta-campo no Rio Grande do Sul. Ciência \& Ambiente, Santa Maria, n. 24, p. 109-118, 2002.

REITZ, R. Vegetação da zona marítima de Santa Catarina. Sellowia, Itajaí, v. 13, p. 17-115, 1961.

SUGUIO, K.; TESSLER, M. G. Planícies de cordões litorâneos quaternários do Brasil: origem e nomenclatura. In: LACERDA, L. D; ARAÚJO, D. S. D.; CERQUEIRA, R.; TURCQ, B. (Eds). Restingas: Origem, Estrutura e Processos. Niterói: CEUFF, 1984. p. 15-26. 